\title{
Vagus Nerve Stimulation: Rapid versus Slow Cycling in a Laboratory Model
}

\author{
Robert S. Fisher ${ }^{1}$
}

Published online: 16 May 2016

(C) The American Society for Experimental NeuroTherapeutics, Inc. 2016

Key Words Vagus nerve stimulation · neurostimulation . stimulation parameters $\cdot$ hippocampal field potentials

Timing of neurostimulation has historically differed for epilepsy and movement disorders, with the former being intermittent and the latter being continuous. Reasons for this variation are uncertain. Studies by Agnew et al. [1] on peripheral nerves suggested that tissue was less stressed by intermittent electrical stimulation, although this may not be the case for clinical neurostimulation within safe parameters. The original neurocybernetic vagus nerve stimulation (VNS) system was designed to allow a range of on and off periods from $5 \mathrm{~min}$ to $3 \mathrm{~h}[2,3]$, "believed to be adequate for recovery of the nerve from the effects of the previous stimulation," with reference to Agnew and McCreery [4]. In a study by Zabara [5] of canine strychnine-induced seizures, VNS for 4 min attenuated seizures for 16 min after stimulation. A "rough rule of thumb" was suggested that seizures are suppressed for a time period 4fold the duration of the stimulation. If this rule extended to the clinical trials, then the suppression induced by $30 \mathrm{~s}$ of VNS would be expected to last for $2 \mathrm{~min}$. The initial clinical trial employed a schedule of $30 \mathrm{~s}$ stimulation on, followed by 5 min off, on a regular clock cycle [6].

When the therapeutic response to VNS is suboptimal after 3 months of therapy, increasing the duty cycle (ratio of time on to time off) has been recommended [7]. Benefits of such a

Robert S. Fisher

robert.fisher@stanford.edu

1 Department of Neurology, Stanford Neuroscience Health Center, Room 2852, 213 Quarry Road, MC 5979, Palo Alto, CA 94304-5979, USA

change are individual and anecdotal. A review of response to different parameter settings observed a median reduction of seizure frequency of $21 \%$ at 3 months of stimulation versus $39 \%$ at 12 months when off time was reduced to $<1.1 \mathrm{~min}$ [8]. Other cycle times did not experience that degree of improvement from 3 to 12 months, but those other cycle times started at a higher level of improvement at 3 months. DeGiorgio et al. [9] randomized 61 subjects to 1 of 3 regimens (seconds on/seconds off): 7/18, 30/30, 30/180. Median seizure reductions were, respectively, $22 \%, 26 \%$, and $29 \%$ (nonsignificantly different). A VNS registry of 269 patients found no differences with standard or rapid cycling, or when changing from standard to rapid cycling [10]. Therefore, the evidence for benefit of rapid cycling of VNS is limited. The latest generation of stimulator can deliver stimulation in response to a preprogrammed degree of ictal tachycardia, thereby providing a type of responsive rapid cycling $[11,12]$.

When optimal stimulation parameters and the mechanisms underlying them are unknown, controlled experiments in a laboratory can be useful, and such an experiment is provided by the article by Larsen et al. [13] in this issue, entitled "Vagus nerve stimulation applied with a rapid cycle has more profound influence on hippocampal electrophysiology than standard cycle." The investigators implanted stimulating electrodes in rat perforant path (PP), which is afferent to dentate gyrus of hippocampus, and recording field [electroencephalography (EEG)] electrodes in dentate gyrus. Spontaneous dentate EEG and EEG evoked by PP stimulation were studied under different conditions of vagus nerve activation. VNS decreased the rise slope of the field excitatory postsynaptic potential and increased the latency of the population spike, while also increasing the amplitude of the population spike. VNS also reduced thetaband power in dentate and partially uncoupled theta-gamma synchrony. Taken collectively, these findings can be interpreted as decreasing hippocampal excitability and network synchrony, 
which could be useful for attenuating seizures. Rapid cycling at $7 \mathrm{~s}$ on, $18 \mathrm{~s}$ off influenced hippocampal electrophysiology more than slow cycling did at $30 \mathrm{~s}$ on, $300 \mathrm{~s}$ off. The rapid cycling was more influential, even at lower stimulating currents.

The main value of the Larsen et al. study [13] is further development of a controlled experimental bioassay to test the utility of different stimulation parameters. The challenge will be in translating findings from the laboratory model to the clinic. Rats are not patients and evoked potentials are not spontaneous seizures. Nevertheless, this is a start. VNS has been around for many years; it is past time to develop methods to optimize this therapy.

Required Author Forms Disclosure forms provided by the authors are available with the online version of this article.

\section{References}

1. Agnew WF, McCreery DB, Yuen TG, Bullara LA. Histologic and physiologic evaluation of electrically stimulated peripheral nerve: considerations for the selection of parameters. Ann Biomed Eng 1989;17:39-60.

2. Terry R, Tarver WB, Zabara J. An implantable neurocybernetic prosthesis system. Epilepsia 1990;31(Suppl 2):S33-S37.

3. Terry RS, Tarver WB, Zabara J. The implantable neurocybernetic prosthesis system. Pacing Clin Electrophysiol 1991;14:86-93.
4. Agnew WF, McCreery DB. Considerations for safety with chronically implanted nerve electrodes. Epilepsia 1990;31(Suppl 2):S27S32.

5. Zabara J. Inhibition of experimental seizures in canines by repetitive vagal stimulation. Epilepsia 1992;33:1005-1012.

6. Ben-Menachem E, Mañon-Espaillat R, Ristanovic R, et al. Vagus nerve stimulation for treatment of partial seizures: 1. A controlled study of effect on seizures. First International Vagus Nerve Stimulation Study Group. Epilepsia 1994;35:616-626.

7. Heck C, Helmers SL, DeGiorgio CM. Vagus nerve stimulation therapy, epilepsy, and device parameters: scientific basis and recommendations for use. Neurology 2002;59(6 Suppl 4):S31-S37.

8. DeGiorgio CM, Thompson J, Lewis P, et al. Vagus nerve stimulation: analysis of device parameters in 154 patients during the longterm XE5 study. Epilepsia 2001;42:1017-1020.

9. DeGiorgio C, Heck C, Bunch S, et al. Vagus nerve stimulation for epilepsy: randomized comparison of three stimulation paradigms. Neurology 2005;65:317-319.

10. Labar D. Vagus nerve stimulation for 1 year in 269 patients on unchanged antiepileptic drugs. Seizure 2004;13:392-398.

11. Fisher RS, Afra P, Macken M, et al. Automatic Vagus Nerve Stimulation Triggered by Ictal Tachycardia: Clinical Outcomes and Device Performance-The U.S. E-37 Trial. Neuromodulation 2016;19:188-195.

12. Boon P, Vonck K, van Rijckevorsel K, et al. A prospective, multicenter study of cardiac-based seizure detection to activate vagus nerve stimulation. Seizure 2015;32:52-61.

13. Larsen et al. Vagus nerve stimulation applied with a rapid cycle has more profound influence on hippocampal electrophysiology than standard cycle. Neurotherapeutics 2016. 